\title{
Suppressive effects of exogenous regucalcin on the proliferation of human pancreatic cancer MIA PaCa-2 cells in vitro
}

\author{
MASAYOSHI YAMAGUCHI $^{1}$ and TOMIYASU MURATA ${ }^{2}$ \\ ${ }^{1}$ Department of Hematology and Medical Oncology, Emory University School of Medicine, Atlanta, GA, USA; \\ ${ }^{2}$ Department of Analytical Neurosciences, Faculty of Pharmaceutical Sciences, Meijo University, Nagoya, Aichi, Japan
}

Received January 16, 2015; Accepted April 1, 2015

DOI: 10.3892/ijmm.2015.2164

\begin{abstract}
Regucalcin plays a pivotal role as a suppressor protein in signal transduction in various types of cells and tissues. The regucalcin gene is localized on the $\mathrm{X}$ chromosome. and its expression has been shown to be suppressed in various types of tumor tissue in animal and human subjects, suggesting a potential role of regucalcin in carcinogenesis. This study was undertaken to determine the effects of exogenous regucalcin on the proliferation of cloned human pancreatic cancer MIA PaCa-2 cells in vitro. The proliferation of the MIA PaCa-2 cells was suppressed following culture with regucalcin (0.01-10 $\mathrm{nM}$ ). Such an effect was also observed in pancreatic cancer Pt45P1 cells, that highly expressed tissue factor (high TF), or Pt45P1 cells, that highly expressed alternativly spliced variants of tissue factor (asTF). In the MIA PaCa- 2 cells, the suppressive effects of regucalcin on cell proliferation were not enhanced either in the presence of tumor necrosis factor- $\alpha$ (TNF- $\alpha$ ), or in the presence of Bay K 8644 , PD98059, staurosporine, wortmannin or 5,6-dichloro-1- $\beta$-D -ribofuranosylbenzimidazole (DRB). However, this was not the case for gemcitabine, which was shown to suppress cell proliferation. Exogenous regucalcin did not cause apoptotic cell death in the MIA PaCa-2 cells in vitro. These findings demonstrate that exogenous regucalcin exerts suppressive effects on the proliferation of human pancreatic MIA PaCa-2 cells and that these effects are mediated through the inhibition of various signaling pathways related to nuclear factor- $\kappa \mathrm{B}$ $(\mathrm{NF}-\kappa \mathrm{B})$, extracellular signal-regulated kinase (ERK), protein kinase $\mathrm{C}$, calcium signaling, phosphatidylinositol 3-kinase (PI3K) or nuclear transcription activity in vitro. Our data suggest that exogenous regucalcin exerts suppressive effects on the proliferation of human pancreatic cancer cells.
\end{abstract}

Correspondence to: Masayoshi Yamaguchi, Department of Hematology and Medical Oncology, Emory University School of Medicine, 1365-C Clifton Road NE, Atlanta, GA 30322, USA

E-mail: yamamasa1155@yahoo.co.jp

Key words: regucalcin, cell proliferation, pancreatic cancer, MIA PaCa-2 cells, Pt45P1 cells, apoptosis, cell signaling, carcinogenesis

\section{Introduction}

Regucalcin was discovered in 1978 as a novel calcium-regulatory protein (1-4), and has been demonstrated to play a multifunctional role in the regulation of various types of cells and tissues (5-7). The regucalcin gene ( $r g n)$ is localized on the $\mathrm{X}$ chromosome and has been identified in over 15 species in vertebrates and invertebrates (7-11). Regucalcin gene expression is regulated by various transcription factors, including activator protein-1 (AP-1), nuclear factor I-A1 (NF1-A1), regucalcin gene promoter region-related protein (RGPR-p117) and $\beta$-catenin, which are modulated through intracellular signaling factors related to the phosphorylation and dephosphorylation of various proteins in the cytoplasm and nucleus in vitro (11). Regucalcin is expressed in various types of cells and tissues. Regucalcin gene expression is regulated by various hormonal factors $(11,12)$.

Regucalcin, which is present in the cytoplasm, is translocated to the nucleus in various cell types dependent on the activation of calcium signaling (13). Regucalcin plays a role in the maintenance of intracellular calcium homeostasis, the inhibition of various protein kinases, protein phosphatases and protein synthesis in the cytoplasm and nucleus, as well as in the nuclear gene expression and DNA and RNA syntheses in various cell types $(5-7,13)$. Moreover, regucalcin has been shown to suppress cell proliferation and apoptotic cell death, which is mediated through various signaling factors $(14,15)$. Regucalcin has been suggested to play a physiological role in maintaining cell homeostasis as a regulatory protein in intracellular signaling systems $(14,15)$.

Regucalcin has been demonstrated to play a pathophysiological role in metabolic disorders and diseases (16-19). Of note, regucalcin has also been shown to be involved in carcinogenesis (19). The gene and protein expression of regucalcin has been found to be suppressed in various types of tumor tissue in mammalian models and human subjects in vivo $(19,20)$. It has also been shown that regucalcin gene expressionis downregulated during the development of carcinogenesis $(14,19)$. The overexpression of endogenous regucalcin has been shown to suppress the proliferation of cloned rat hepatoma H4-II-E cells in vitro, in which regucalcin gene expression is downregulated (21).

Moreover, regucalcin has been suggested to play a role as a suppressor protein in human carcinogenesis $(19,20)$. The present study was undertaken in an effort to determine whether exogenous regucalcin exerts a suppressive effect on the proliferation of pancreatic cancer cells in vitro. We found 
that exogenous regucalcin suppressed the in vitro proliferation of pancreatic cancer MIA $\mathrm{PaCa}-2$ cells, which are resistant to radiation therapy; however, regucalcin did not have an effect on apoptotic cell death.

\section{Materials and methods}

Materials (reagents). Dulbecco's modified Eagle's medium (DMEM) with $4.5 \mathrm{~g} / 1$ glucose, L-glutamine and sodium pyruvate and antibiotics (penicillin and streptomycin) were purchased from Invitrogen Corp. (Carlsbad, CA, USA). Fetal bovine serum (FBS) was from HyClone (Logan, UT, USA). Tumor necrosis factor- $\alpha$ (TNF- $\alpha$ ) was from R\&D Systems (Minneapolis, MN, USA). PD98059 [an extracellular signal-regulated kinase (ERK) inhibitor], staurosporine (an inhibitor of protein kinase C), Bay K 8644 (an agonist of $\mathrm{Ca}^{2+}$ influx in cells), wortmannin [an inhibitor of phosphatidylinositol 3-kinase (PI3K)] or 5,6-dichlor o-1- $\beta$-D-ribofuranosylbenzimidazole (DRB; an inhibitor of transcriptional activity with RNA polymerase II inhibition) and all other reagents were purchased from Sigma-Aldrich (St. Louis, MO, USA) unless otherwise specified. Gemcitabine was obtained from Hospira, Inc. (Lake Forest, IL, USA), and it was diluted in phosphate-buffered saline (PBS). All procedure and protocols for the use of the rat livers were approved by the Institutional Animal Care and Use Committee at Emory University.

Regucalcin. Regucalcin was isolated from rat liver cytosol, as previously described (1). The livers were perfused with Tris- $\mathrm{HCl}$ buffer (pH 7.4), containing $100 \mathrm{mM}$ Tris, $120 \mathrm{mM} \mathrm{NaCl}, 4 \mathrm{mM}$ $\mathrm{KCl}$, cooled to $4^{\circ} \mathrm{C}$. The livers were then removed, cut into small sections, suspended 1:4 (w/v) in Tris-HCl buffer ( $\mathrm{pH}$ 7.4) and homogenized in a Potter-Elvehjem homogenizer with a Teflon pestle, as previously described (1). The homogenate was spun at 5,500 $\mathrm{x} \mathrm{g}$ in a refrigerated centrifuge for $10 \mathrm{~min}$, and the supernatant was spun at $105,00 \mathrm{x} g$ for $60 \mathrm{~min}$. The resulting supernatant was isolated to electorophoretic homogeneity by gel filtration on Sephadex G-75 and G-50, followed by ion-exchange chromatography on diethylaminoethyl (DEAE)-cellulose, as previously described (1). The purity of the isolated regucalcin was confirmed using SDS-gel electrophoresis and western blot analysis.

Pancreatic cancer cells. For our experiments, we used pancreatic cancer MIA PaCa-2 cells, Pt45P1 cells with a high expression of tissue factor (high TF) or Pt45P1 cells with a high expression of alternatively spliced variants of tissue factor (asTF) $(22,23)$. These human pancreatic cancer cell lines were obtained from the American Type Culture Collection (Rockville, MD, USA).

Cell proliferation. Pancreatic cancer MIA PaCa-2, Pt45P1 (high TF) or Pt45P1 (asTF) cells (1x10 $/ \mathrm{ml} /$ well) were cultured using a 24-well plate in DMEM containing 10\% FBS and $1 \%$ penicillin/streptomycin $(\mathrm{P} / \mathrm{S})$ in the presence or absence of regucalcin $(0.01,0.1,0.5,1$ or $10 \mathrm{nM})$ for $1,2,3$ and 7 days, as previously described (21). In separate experiments, the cells $\left(1 \times 10^{5} / \mathrm{ml} /\right.$ well $)$ were cultured in DMEM containing $10 \% \mathrm{FBS}$ and $1 \% \mathrm{P} / \mathrm{S}$ in the presence of TNF- $\alpha(1 \mathrm{ng} / \mathrm{ml})$, Bay K $8644(1 \mu \mathrm{M})$, PD98059 $(1 \mu \mathrm{M})$, staurosporine $(0.1 \mu \mathrm{M})$, wortmannin $(1 \mu \mathrm{M})$ or DRB $(1 \mu \mathrm{M})$ for 3 days. Following culture, the cells were detached from each culture dish to determine the cell number.

Apoptotic cell death. The pancreatic cancer MIA PaCa-2 or Pt45P1 (high TF) cells (1x10 $/ \mathrm{ml} /$ well) were cultured in a 24-well plate in DMEM containing 10\% FBS and 1\% P/S in the absence of regucalcin for 7 days until they reached confluency (85-95\%). Subsequently, the cells were cultured in the presence or absence of regucalcin $(0.1,1$ or $10 \mathrm{nM})$ with or without gemcitabine $(10-1,000 \mathrm{nM})$ for 7 days, as previously described (15). Following culture, the cells were detached from each culture dish to determine the cell number.

Cell counting. Following trypsinization of each of the culture dishes using $0.2 \%$ trypsin plus $0.02 \%$ EDTA in $\mathrm{Ca}^{2+} / \mathrm{Mg}^{2+}$-free PBS for $2 \mathrm{~min}$ at $37^{\circ} \mathrm{C}$, the detached cells from each dish were collected following centrifugation. The cells were resuspended in PBS solution and then stained with eosin. The cell numbers were counted under a microscope using a hemocytometer (Sigma-Aldrich). For each dish, the average of two countings was used. Cell numbers are presented as the number of cells per well in each plate.

Statistical analysis. Statistical significance was determined using GraphPad InStat software version 3 for Windows XP (GraphPad Software, Inc., La Jolla, CA, USA). Multiple comparisons were performed by one-way analysis of variance (ANOVA) with the Tukey-Kramer multiple comparisons post-hoc test for parametric data. A value of $\mathrm{P}<0.05$ was considered to indicate a statistically significant difference.

\section{Results}

To determine the effects of exogenous regucalcin on the proliferation of human pancreatic cancer cells, we used MIA PaCa-2 cells, which are resistant to radiation. The MIA PaCa-2 cells were cultured in the presense of exogenous regucalcin (0.01-10 nM) for 1-7 days (Fig. 1). The cell numbers increased in a time-dependent manner (Fig. 1). The addition of exogenous regucalcin diminished the increase in cell number (Fig. 1), thus suggesting that cell proliferation was is suppressed by physiological concentrations of serum regucalcin (24).

Subsequently, in order to determine the suppressive effects of exogenous regucalcin on the proliferation of other human pancreatic cancer cells, we used Pt45P1 cells, which highly expressed tissue factor (high TF; Fig. 2A) or which highly expressed alternatively spliced variants of tissue factor (asTF; Fig. 2B) in vitro. These cells were cultured for 7 days in the presence or absence of regucalcin $(0.1,1$ or $10 \mathrm{nM})$. The addition of exogenous regucalcin had a suppressive effect on the Pt45P1 cells (high TF and asTF;Fig. 2).

The suppressive effects of exogenous regucalcin on the proliferation of the pancreatic cancer MIA PaCa- 2 cells were compared with the effects of other factors that have been shown to decrease cell proliferation. As shown in Fig. 3, the suppressive effects of exogenous regucalcin $(1 \mathrm{nM})$ on the proliferation of MIA PaCa-2 cells were not enhanced in the presence of $\mathrm{TNF}-\alpha(1 \mathrm{ng} / \mathrm{ml})$, an enhancer of nuclear factor- $\mathrm{B}(\mathrm{NF}-\kappa \mathrm{B})$ signaling (25), or in the presence of Bay K $8644(1 \mu \mathrm{M})$, an agonist of $\mathrm{Ca}^{2+}$ influx in cells (26). 

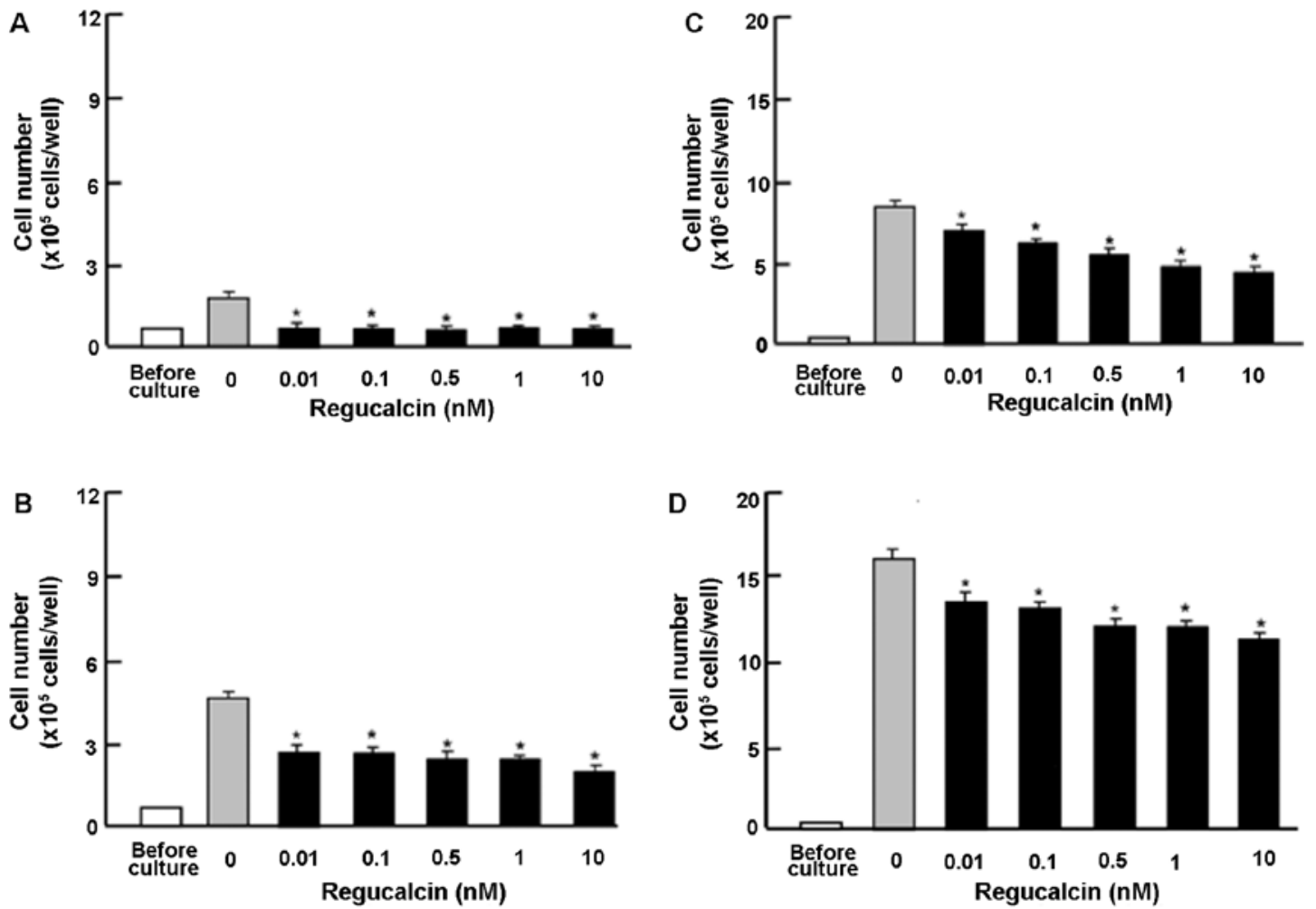

Figure 1. Exogenous regucalcin suppresses the proliferation of pancreatic cancer MIA PaCa-2 cells in vitro. Cells were cultured in Dulbecco's modified Eagle's medium (DMEM) in the presence or absence of regucalcin (0.01-10 nM) for (A) 1, (B) 2, (C) 3 or (D) 7 days. Following culture, the number of attached cells on the dish was counted. Data are presented as the means \pm SD of 2 replicate wells per data set using different dishes and cell preparations. ${ }^{*} \mathrm{P}<0.001$ vs. control (grey bar), as shown by one-way ANOVA and the Tukey-Kramer post-hoc test.
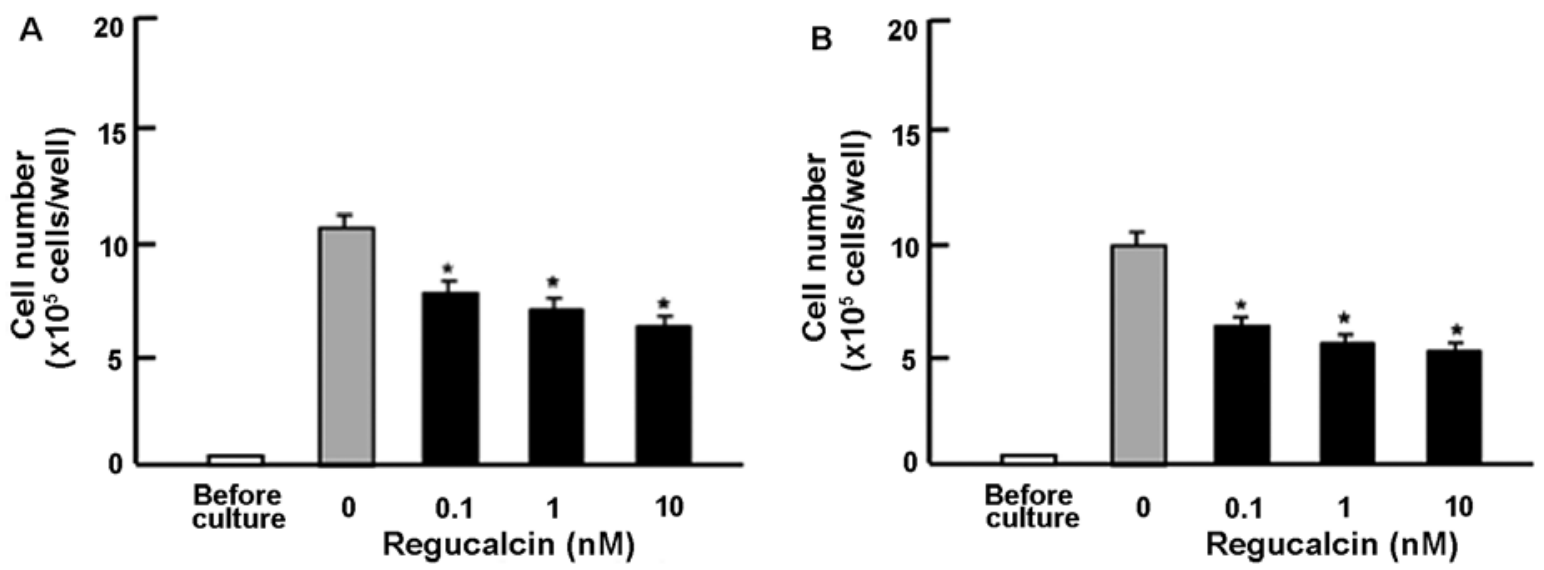

Figure 2. Exogenous regucalcin suppresses the proliferation of pancreatic cancer Pt45P1 cells in vitro. (A) Pt45P1 cells with a high expression of tissue factor (TF), (B) Pt45P1 cells with a high expression of alternatively spliced variants of TF (asTF). Cells were cultured for 7 days in the presence $(0.1,1$ or $10 \mathrm{nM})$ or absence of regucalcin. Following culture, the number of attached cells on the dish was counted. Data are presented as the means \pm SD of 2 replicate wells per data set using different dishes and cell preparations. ${ }^{*} \mathrm{P}<0.001$ vs. control (grey bar), as shown by one-way ANOVA and the Tukey-Kramer post-hoc test.

Subsequently, we determined whether the suppressive effects of exogenous regucalcin on cell proliferation involve intracellular signaling pathways. The results revealed that the suppressive effects of exogenous regucalcin on cell proliferation were not enhanced in the presence of PD98059 $(1 \mu \mathrm{M})$, an ERK inhibitor (27), or staurosporine $(0.1 \mu \mathrm{M})$, an inhibitor of protein kinase C (28) (Fig. 4A). Moreover, the suppressive effects of regucalcin on cell proliferation were not enhanced in the presence of wortmannin $(1 \mu \mathrm{M})$, an inhibitor of PI3K (29), or
DRB $(1 \mu \mathrm{M})$, an inhibitor of transcriptional activity with RNA polymerase II inhibition (30) (Fig. 4B).

Moreover, the effects of exogenous regucalcin were compared with those of gemcitabine, an antitumor agent that induces nuclear DNA damage (31). The suppressive effects of regucalcin on the proliferation of pancreatic cancer cells were examined in the presence of gemcitabine. Culture with gemcitabine $(100-1,000 \mathrm{nM})$ suppressed the proliferation of the MIA PaCa-2 cells (Fig. 5A). The suppressive effects of regucalcin $(1 \mathrm{nM})$ on the proliferation 


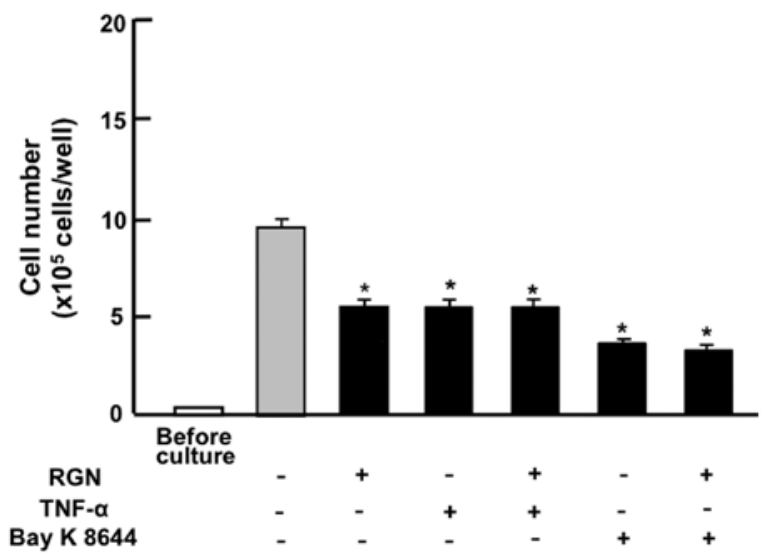

Figure 3. Suppressive effects of regucalcin on the proliferation of pancreatic cancer MIA PaCa- 2 cells in the presence of tumor necrosis factor- $\alpha$ (TNF- $\alpha$ ) or Bay K 8644 (an agonist of $\mathrm{Ca}^{2+}$ influx in cells) in vitro. Cells were cultured for 3 days in the presence of regucalcin $(1 \mathrm{nM})$ with or without TNF- $\alpha(1 \mathrm{ng} /$ $\mathrm{ml})$ or Bay K $8644(1 \mu \mathrm{M})$. Following culture, the number of attached cells on the dish was counted. Data are presented as the means \pm SD of 2 replicate wells per data set using different dishes and cell preparations. ${ }^{\mathrm{P}}<0.001$ vs. control (grey bar), as shown by one-way ANOVA and the Tukey-Kramer post-hoc test. RGN, regucalcin.
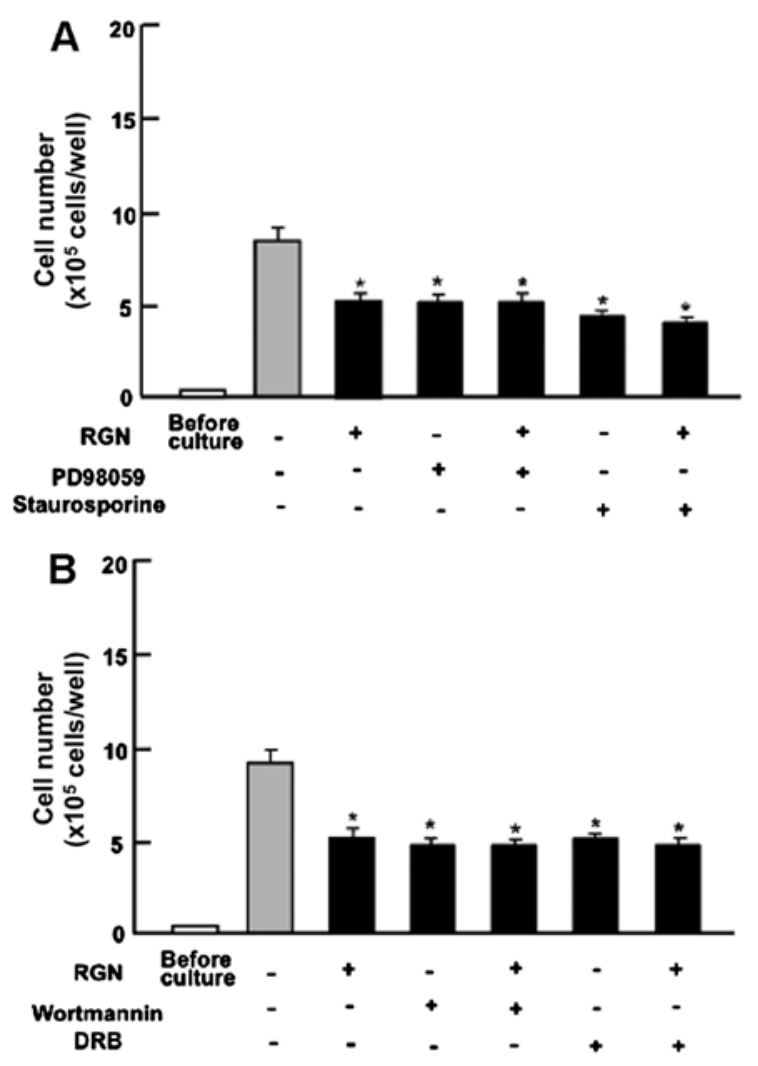

Figure 4. Suppressive effects of regucalcin on the proliferation of pancreatic cancer MIA PaCa-2 cells in the presence of various inhibitors in vitro (A) Cells were cultured for 3 days in the presence of regucalcin $(1 \mathrm{nM})$ with or without PD98059 (an ERK inhibitor, $1 \mu \mathrm{M}$ ) or staurosporine (an inhibitor of protein kinase $\mathrm{C}, 0.1 \mu \mathrm{M}$ ). (B) Cells were cultured for 3 days in the presence of regucalcin $(1 \mu \mathrm{M})$ with or without wortmannin (an inhibitor of PI3K, $1 \mu \mathrm{M}$ ) or 5,6-dichloro-1- $\beta$-D-ribofuranosylbenzimidazole (DRB; an inhibitor of transcriptional activity with RNA polymerase II inhibition; $1 \mu \mathrm{M})$. Following culture, the number of attached cells on dish was counted. Data are presented as the means \pm SD of 2 replicate wells per data set using different dishes and cell preparations. ${ }^{*} \mathrm{P}<0.001$ vs. control (grey bar), as shown by one-way (ANOVA and the Tukey-Kramer post-hoc test. RGN, regucalcin.
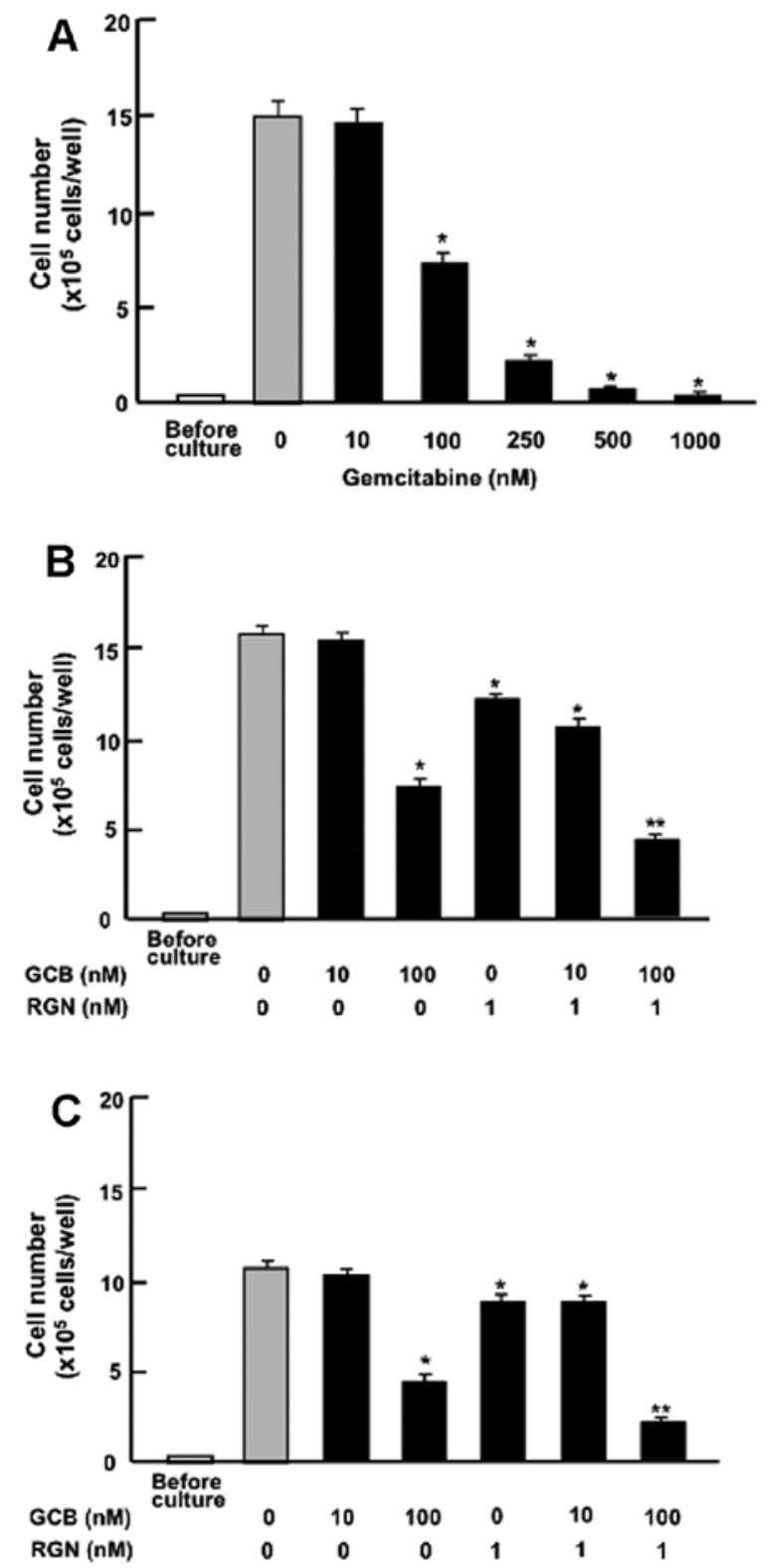

Figure 5. Suppressive effects of regucalcin on the proliferation of pancreatic cancer MIA PaCa-2 cells in the presence of gemcitabine (GCB) in vitro. (A) Cells were cultured for 7 days in the presence of GCB (10-1,000 nM). (B) MIA PaCa-2 cells were cultured for 7 days in the presence of regucalcin $(1 \mathrm{nM})$ with or without GCB (10 or $100 \mathrm{nM})$. (C) Pancreatic cancer Pt45P1 cells [which highly express tissue factor (high TF)] were cultured for 7 days in the presence of regucalcin $(1 \mathrm{nM})$ with or without GCB $(10$ or $100 \mathrm{nM})$. Following culture, the number of attached cells on the dish was counted. Data are presented as the means \pm SD of 2 replicate wells per data set using different dishes and cell preparations. ${ }^{*} \mathrm{P}<0.00$ vs. control (grey bar); ${ }^{* *} \mathrm{P}<0.001$ vs. GCB $(100 \mathrm{nM})$ alone, as shown by one-way ANOVA and the Tukey-Kramer post-hoc test. RGN, regucalcin.

of the MIA PaCa-2 cells were also observed in the presence of low concentrations of gemcitabine $(10 \mathrm{nM})$, which did not have a significant effect on cell proliferation (Fig. 5B). However, the suppressive effects of regucalcin $(1 \mathrm{nM})$ on cell proliferation were significantly enhanced in the presence of higher/high concentrations of gemcitabine $(100 \mathrm{nM})$ that had a suppressive effect on cell proliferation (Fig. 5B). A similar effect was also produced by treatment with $1 \mathrm{nM}$ regucalcin in combination with $100 \mathrm{nM}$ gemcitabine in the Pt45P1 (high TF) cells (Fig. 5C). 

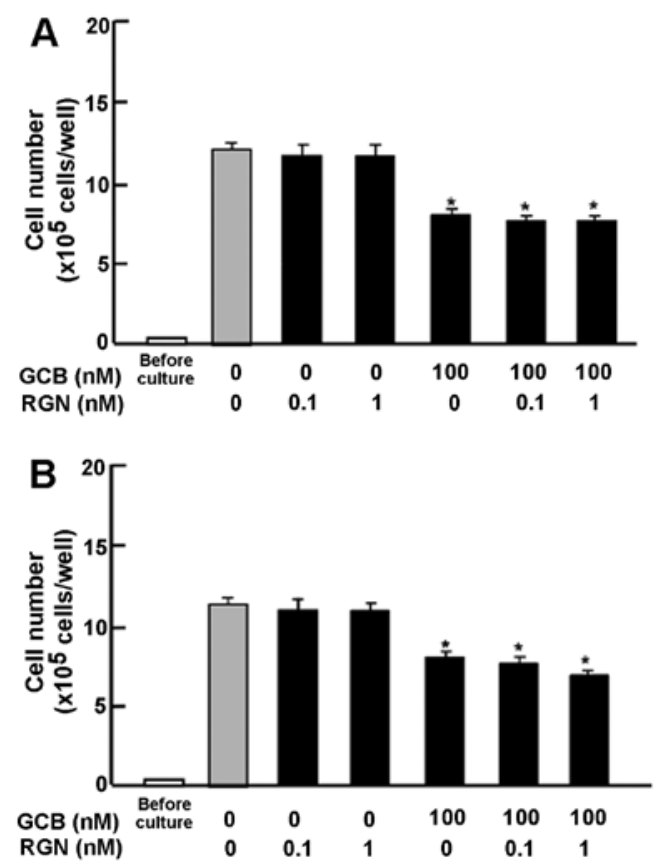

Figure 6. Effect of regucalcin on apoptotic cell death in pancreatic cancer MIA PaCa-2 cells in the presence or absence of gemcitabine (GCB) in vitro. Cells were cultured for 7 days until reaching confluency, and then the cells were cultured for an additional (A) 3 or (B) 7 days in the presence of regucalcin $(0.1$ or $1 \mathrm{nM})$ with or without GCB $(100 \mathrm{nM})$. Following culture, the number of attached cells on the dish was counted. Data are presented as the means \pm SD of 2 replicate wells per data set using different dishes and cell preparations. " $\mathrm{P}<0.001$ vs. control (grey bar), as shown by one-way ANOVA and the Tukey-Kramer post-hoc test. RGN, regucalcin.

The effects of regucalcin on apoptotic cell death in the pancreatic cancer MIA PaCa- 2 cells were also determined. The cells were cultured for 7 days until reaching confluency, and the cells were then cultured for an additional 3 days (Fig. 6A) or 7 days (Fig. $6 \mathrm{~B})$ in the presence of regucalcin $(0.1$ or $1 \mathrm{nM})$ with or without gemcitabine $(100 \mathrm{nM})$. The addition of exogenous regucalcin did not cause apoptotic cell death, whereas culture with gemcitabine for 3 or 7 days caused apoptotic cell death (Fig. 6). This effect was not significantly affected in the presence of regucalcin (Fig. 6).

\section{Discussion}

Previous studies have demonstrated that regucalcin plays a potential role as a suppressor of cell proliferation and carcinogenesis $(14,19)$. Regucalcin gene expression has been found to be downregulated in the tumor tissues of human subjects (20) and human cancer cells $(18,32)$. The present study demonstrated that the proliferation of human pancreatic cancer MIA PaCa-2 and Pt45P1 (high TF and asTF) cells was suppressed by the addition of exogenous regucalcin at physiological concentrations (24), and that regucalcin did not have an effect on apoptotic cell death in vitro. To the best of our knowledge, this is the first time that regucalcin was shown to play a critical role in the suppression of human pancreatic cancer cell proliferation.

The overexpression of endogenous regucalcin has been shown to suppress the proliferation of cloned rat hepatoma H4-II-E cells in vitro $(14,18,21)$. The overexpression of endogenous regucalcin has been demonstrated to cause $\mathrm{G} 1$ and $\mathrm{G} 2 / \mathrm{M}$ phase cell cycle arrest in rat hepatoma H4-II-E cells (33) and in rat normal kidney NRK52E cells (34). The suppressive effects of endogenous regucalcin on cell proliferation are mediated through the suppression of the activities of $\mathrm{Ca}^{2+}$ signaling-dependent protein kinases, protein phosphatases and $\mathrm{PI} 3 \mathrm{~K}$, which are involved in various signaling pathways $(14,18)$. The overexpression of endogenous regucalcin has been shown to suppress $c$-myc, Ha-ras, $c$-jun and $c h k 2$ mRNA expression or enhance $p 53$ and $R b$ mRNA expression $(14,19,35,36)$. Moreover, regucalcin has been found to suppress cytoplasmic protein synthesis and nuclear DNA and RNA synthesis $(13,14)$. Thus, endogenous regucalcin exerts suppressive effects on cell proliferation through multifunctional pathways in rat normal and cancer cells.

In addition, regucalcin has been shown to bind to the plasma membranes of rat liver in vitro (37). It is possible that exogenous regucalcin may bind to the plasma membranes of human pancreatic cancer MIA PaCa-2 cells and may thus regulate the intracellular signaling pathways that suppress cell proliferation. Our results revealed that the suppressive effects of regucalcin on the proliferation of pancreatic cancer MIA $\mathrm{PaCa}-2$ cells were not enhanced either in the presence of TNF- $\alpha$, an enhancer of $\mathrm{NF}-\kappa \mathrm{B}$ signaling (25), Bay $\mathrm{K} 8644$, an agonist of $\mathrm{Ca}^{2+}$ entry in cells (26), PD98059, an ERK inhibitor (27), staurosporine, an inhibitor of calcium-dependent protein kinase $C$ (28), or in the presence of wortmannin, an inhibitor of PI3K (29). Thus, the suppressive effects of exogenous regucalcin on the proliferation of pancreatic cancer MIA $\mathrm{PaCa}-2$ cells were not modulated in the presence of various inhibitors that regulate intracellular signaling pathways related to cell proliferation in vitro. These findings support the view that the suppressive effects of exogenous regucalcin on cell proliferation are mediated through the inhibition of various intracellular signaling pathways (including NF- $\mathrm{KB}$, calcium, ERK, protein kinase $\mathrm{C}$, and PI3K) that are related to the proliferation of human pancreatic cancer MIA PaCa- 2 cells.

Moreover, the results of this study demonstrated that the suppressive effects of regucalcin on cell proliferation were not enhanced in the presence of DRB, an inhibitor of transcriptional activity with RNA polymerase II inhibition (30). Intracellular signals for exogenous regucalcin, which are bound receptors on the plasma membranes of pancreatic cancer cells, may be transmitted into the nucleus to suppress transcriptional regulation and regulate the nuclear function of human pancreatic cancer MIA PaCa-2 cells.

However, the suppressive effects of exogenous regucalcin on the proliferation of MIA PaCa- 2 cells were enhanced in the presence of gemcitabine, an antitumor agent that induces nuclear DNA damage (32). Exogenous regucalcin did not induce apoptotic cell death in human pancreatic cancer MIA PaCa-2 cells in vitro, supporting the view that regucalcin does not have a promoting effect on apoptosis. Thus, the suppressive effects of exogenous regucalcin on the proliferation of human pancreatic cancer MIA PaCa-2 cells were independent of the induction of apoptosis. Exogenous regucalcin did not enhance the effects of gemcitabine on the induction of apoptosis. The mode of action of exogenous regucalcin in suppressing cell proliferation may differ from that of gemcitabine. However, the combination of exogenous regucalcin and gemcitabine may be a useful tool in enhancing the antitumor effects on human pancreatic cancer cells. 
In conclusion, in this study, we demonstrated that exogenous regucalcin had a significant suppressive effect on the proliferation of human pancreatic cancer MIA PaCa-2 cells in vitro, suggesting a critical role for regucalcin as a novel cytokine that suppresses cell proliferation.

\section{References}

1. Yamaguchi $\mathrm{M}$ and Yamamoto T: Purification of calcium binding substance from soluble fraction of normal rat liver. Chem Pharma Bull (Tokyo) 26: 1915-1918, 1978.

2. Yamaguchi M and Sakurai T: Inhibitory effect of calcium-binding protein regucalcin on $\mathrm{Ca}^{2+}$-activated DNA fragmentation in rat liver nuclei. FEBS Lett 279: 281-284, 1991.

3. Shimokawa $\mathrm{N}$ and Yamaguchi M: Molecular cloning and sequencing of the cDNA coding for a calcium-binding protein regucalcin from rat liver. FEBS Lett 327: 251-255, 1993.

4. Misawa $\mathrm{H}$ and Yamaguchi $\mathrm{M}$ : The gene of $\mathrm{Ca}^{2+}$-binding protein regucalcin is highly conserved in vertebrate species. Int $\mathrm{J}$ Mol Med 6: 191-196, 2000.

5. Yamaguchi M: Role of regucalcin in calcium signaling. Life Sci 66: 1769-1780, 2000.

6. Yamaguchi M: Role of regucalcin in maintaining cell homeostasis and function (Review). Int J Mol Med 15: 371-389, 2005.

7. Yamaguchi M: Regucalcin and cell regulation: role as a suppressor in signal transduction. Mol Cell Biochem 353: 101-137, 2011.

8. Shimokawa N, Matsuda Y and Yamaguchi M: Genomic cloning and chromosomal assignment of rat regucalcin gene. Mol Cell Biochem 151: 157-163, 1995.

9. Thiselton DL, McDowall J, Brandau O, Ramser J, d'Esposito F, Bhattacharya SS, Ross MT, Hardcastle AJ and Meindl M: An integrated, functionally annotated gene map of the DXS8026-ELK1 internal on human Xp11.3-Xp11.23: Potential hotspot for neurogenetic disorders. Genomics 79: 560-572, 2002.

10. Yamaguchi M, Makino R and Shimokawa N: The 5' end sequences and exon organization in rat regucalcin gene. Mol Cell Biochem 165: 145-150, 1996.

11. Yamaguchi M: The transcriptional regulation of regucalcin gene expression. Mol Cell Biochem 346: 147-171, 2011.

12. Yamaguchi M: Hormonal regulation of regucalcin gene expression: Involvement in cell metabolism. Horm Stud 1: 1, 2013.

13. Yamaguchi M: Role of regucalcin in cell nuclear regulation: Involvement as a transcription factor. Cell Tissue Res 354: 331-341, 2013.

14. Yamaguchi M: Suppressive role of regucalcin in liver cell proliferation: Involvement in carcinogenesis. Cell Prolif 46: 243-253, 2013.

15. Yamaguchi M:The anti-apoptotic effect of regucalcin is mediated through multisignaling pathways. Apoptosis 18: 1145-1153, 2013.

16. Yamaguchi M: Regucalcin and metabolic disorders: Osteoporosis and hyperlipidemia are induced in regucalcin transgenic rats. Mol Cell Biochem 341: 119-133, 2010.

17. Yamaguchi $M$ and Murata T: Involvement of regucalcin in lipid metabolism and diabetes. Metabolism 62: 1045-1051, 2013.

18. Yamaguchi M: Regucalcin as a potential biomarker for metabolic and neuronal diseases. Mol Cell Biochem 391: 157-166, 2014.

19. Yamaguchi M: Involvement of regucalcin as a suppressor protein in human carcinogenesis: insight into the gene therapy. J Cancer Res Clin Oncol: Sep 18, 2014 (Epub ahead of print).

20. Murata T and Yamaguchi M: Alternatively spliced variants of the regucalcin gene in various human normal and tumor tissues. Int J Mol Med 34: 1141-1146, 2014.
21. Misawa H, Inagaki S and Yamaguchi M: Suppression of cell proliferation and deoxyribonucleic acid synthesis in cloned rat hepatoma H4-II-E cells overexpressing regucalcin. J Cell Biochem 84: 143-149, 2001.

22. Ninomiya I, Yamazaki K, Oyama K, Hayashi H, Tajima H, Kitagawa H, Fushida S, Fujimura T and Ohta T: Pioglitazone inhibits the proliferation and metastasis of human pancreatic cancer cells. Oncol Lett 8: 2709-2714, 2014.

23. Sipos B, Möser S, Kalthoff H, Török V, Löhr M and Klöppel G: A comprehensive characterization of pancreatic ductal carcinoma cell lines: Towards the establishment of an in vitro reseach platform. Virchows Arch 442: 444-452, 2003.

24. Yamaguchi $M$ and Isogai $M$ : Tissue concentration of calcium-binding protein regucalcin in rats by enzyme-linked immunoadsorbent assay. Mol Cell Biochem 122: 65-68, 1993.

25. Lee ZH, Kwack K, Kim KK, Lee SH and Kim HH: Activation of c-Jun N-terminal kinase and activator protein 1 by receptor activator of NF-kappaB. Mol Pharmacol 58: 1536-1545, 2000.

26. Cano-Abad MF, Villarroya M, García AG, Gabilan NH and Lopez MG: Calcium entry through L-type calcium channels causes mitochondrial disruption and chromaffin cell death. J Biol Chem 276: 39695-39704, 2001.

27. Chen S, Wang Y, Ruan W, Wang X and Pan C: Reversing multidrug resistance in hepatocellular carcinoma cells by inhibiting extracellular signal-regulated kinase/mitogen-activated protein kinase signaling pathway activity. Oncol Lett 8: 2333-2339, 2014.

28. Chen QW, Edvinsson and Xu CB: Role of ERK/MAPK in endothelin receptor signaling in human aortic smoth muscle cells. BMC Cell Biol 10: 52, 2009.

29. Serrano-Nascimento C, da Silva Teixeira S, Nicola JP, Nachbar RT, Masini-Repiso AM and Nunes MT: The acute inhibitory effect of iodide excess on sodium/iodide symporter expression and activity involves the PI3K/Akt signaling pathway. Endocrinology 155: 1145-1156, 2014

30. Palangat M, Grass JA, Langelier MF, Coulombe B and Landick R: The RPB2 flap loop of human RNA polymerase II is dispensable for transcription initiation and elongation. Mol Cell Biol 31: 3312-3325, 2011.

31. Tang SC and Chen YC: Novel therapeutic targets for pancreatic cancere. World J Gastroenterol 20: 10825-10844, 2014.

32. Maia C, Santos C, Schmitt F and Socorro S: Regucalcin is underexpressed in human breast and prostate cancers: Effect of sex steroid hormones. J Cell Biochem 107: 667-676, 2009.

33. Yamaguchi $M$ and Daimon Y: Overexpression of regucalcin suppresses cell proliferation in cloned rat hepatoma H4-II-E cells: Involvement of intracellular signaling factors and cell cycle-related genes. J Cell Biochem 95: 1169-1177, 2005.

34. Nakagawa T, Sawada N and Yamaguchi M: Overexpression of regucalcin suppresses cell proliferation of cloned normal rat kidney proximal tubular epithelial NRK52E cells. Int J Mol Med 16: 637-643, 2005.

35. Tsurusaki Y and Yamaguchi M: Overexpression of regucalcin modulates tumor-related gene expression in cloned rat hepatoma H4-II-E cells. J Cell Biochem 90: 619-626, 2003.

36. Tsurusaki $\mathrm{Y}$ and Yamaguchi M: Role of regucalcin in liver nuclear function: Binding of regucalcin to nuclear protein or DNA and modulation of tumor-related gene expression. Int J Mol Med 14: 277-281, 2004.

37. Yamaguchi M, Mori S and Kato S: Calcium-binding protein regucalcin is an activator $\left(\mathrm{Ca}^{2+}-\mathrm{Mg}^{2+}\right)$-adenosine triphosphatase in the plasma membranes of rat liver. Chem Pharm Bull (Tokyo) 36: 3532-3539, 1988. 